\title{
Evidence that novel flavors unconditionally suppress weight gain in the absence of flavor-calorie associations
}

\author{
Benjamin M. Seitz ${ }^{1}$ (D) - Mary E. Flaim ${ }^{1} \cdot$ Aaron P. Blaisdell $^{1}$ \\ Published online: 13 February 2020 \\ (C) The Psychonomic Society, Inc. 2020
}

\begin{abstract}
Beginning with Pavlov (1927), there has been great interest in how associative learning processes affect eating behavior. For instance, flavors can become preferred when paired with calories or, conversely, become aversive when paired with illness. This relationship between flavors and caloric or toxic outcomes has been investigated by a number of theorists. We studied the effect of daily consumption of a flavor that was either paired or unpaired with calories provided by sugar on body weight change and daily food consumption over a 21-day period. Over three experiments, we observed an unanticipated attenuation of weight gain following consumption of flavored liquid solutions, particularly when those solutions were non-caloric. However, we did not find any impact of consuming the flavored liquid solutions on appetite. Given differences in weight gain in the absence of differences in the amount of food consumed, we suggest that unconditioned metabolic responses are elicited to initially novel flavor stimuli, even if those flavors are not followed by caloric outcomes. Potential dieting interventions based on these findings are discussed as is how they inform our understanding of the balance between unconditioned and conditioned responses.
\end{abstract}

Keywords Pavlovian conditioning $\cdot$ Metabolism $\cdot$ Weight gain $\cdot$ Eating $\cdot$ Energy regulation $\cdot$ Learning

\section{Introduction}

The obesity epidemic currently poses a major health (Williams, Mesidor, Winters, Dubbert, \& Wyatt, 2015) and financial (Tremmel, Gerdtham, Nilsson, \& Saha, 2017) threat to societies around the world. Rates of obesity have increased dramatically over the past several decades, with environmental factors being strongly implicated (Apovian, 2016). As food intake and nutrient digestion are necessary factors in weight gain, a deeper understanding of the mechanisms that govern food consumption and metabolic processes is needed in order to address possible interventions to treat obesity.

Beginning with Pavlov (1927), there has been great interest in the role of associative learning in eating behavior, including the regulation of appetite. Food choice and foraging decisions are influenced by associative learning about food cues. Such learning aids in discriminating food from non-food (e.g., as food preferences and aversions), readies digestion (e.g., through the release of digestive

Benjamin M. Seitz

bseitz1@ucla.edu

1 Department of Psychology, University of California, Los Angeles, Los Angeles, CA, USA enzymes), and regulates post-prandial energy regulation (e.g., through insulin signaling). For example, Pavlov (1910) found that the viscosity and amount of saliva that was elicited by the presentation of a food stimulus differed depending on what that food stimulus was. Moreover, he found sham feeding, a procedure in which surgical manipulations prevent chewed and swallowed food from reaching the stomach, lasted longer and resulted in more gastric secretion for meat than for bread (Pavlov, 1910; Smith, 1995).

Many others have attempted to use Pavlovian principles of conditional relationships to understand a variety of aspects related to eating behaviors. For instance, a flavor can serve as a conditioned stimulus (CS) that signals incoming calories that serve as an unconditioned stimulus (US). Once conditioned, flavor CSs tend to be preferred over flavors not associated with calories (Capaldi, 1996; Capaldi, Hunter, \& Lyn, 1997; Sclafani, 2001). In a simple demonstration of this effect, Bolles, Hayward, and Crandall (1981) gave rats access to a flavor paired with flour (CS+) while a different flavor was paired with chalk (CS-; "+" and "-" indicate the presence or absence of the caloric US). Rats had access to both of these mixtures for several days and were then presented with the flavors combined with a mixture of flour and chalk. Rats consumed more of the mixture that contained the CS+ flavor than that 
which contained the CS- flavor. Mehiel and Bolles (1984) conducted a similar experiment in which they paired flavors with either sucrose or saccharin (both sweet tasting) in a solution to rule out the possibility that rats simply were avoiding the potentially aversive chalk. Rats overwhelmingly preferred the solutions containing sucrose over saccharin. Acquired preference for flavors that had been paired with calories has also been demonstrated in humans (Birch, McPhee, Steinberg, \& Sullivan, 1990; Brunstrom \& Mitchell, 2007; but see Brunstrom, 2007, and Yeomans, 2012). The past several decades of research, much of it led by Anthony Sclafani and his colleagues, has demonstrated the complex border parameters and neurobiology that govern flavor-outcome learning (for recent reviews see, Myers, 2018; Sclafani, 2018). For instance, a flavor CS paired with sweetness may become preferred not only because it is reinforced with the caloric outcome (US), but also because it is reinforced with the sweet taste outcome (US). Thus, precise experimental designs and procedures have been developed to dissociate the reinforcing effects of both outcomes, and post-oral hedonic and post-ingestive nutrient outcomes appear to reinforce flavors in dissociable ways (Myers \& Hall, 1998).

While there are many examples of learned flavor preferences, there also exists evidence of unconditioned preferences for certain tastes. Note there is a difference between a taste and a flavor, as a flavor requires an olfactory component (Small \& Prescott, 2005). Also note that we use the term unconditioned instead of innate due to the challenge of proving a response to have occurred in the absence of experiential input (e.g., prenatal and postnatal development). Of course, proving a response to be truly unconditioned is difficult as well because it requires knowledge of all previous encounters with various stimuli. Fortunately, using a rodent model and unique flavors allows for this possibility, hence our use of the term unconditioned. Infant rats demonstrate likings to sweet and moderately salty tastes as well as aversion to bitter and sour tastes (Bartoshuk \& Beauchamp, 1994; Birch, 1999; Myers \& Sclafani, 2006; Vigorito \& Sclafani, 1988). Even fetal rats on day 19 of gestation have shown aversions to lemon infusions and preferences towards milk infusions (note milk has both taste and flavor components) (Smotherman \& Robinson, 1987). While there exists evidence of unconditioned preferences to certain tastes as well as to flavors specific to milk in mammals, whether there are unconditioned preferences and/or responses to non-species relevant flavors is underexplored. By non-relevant, we mean flavors that are not naturally found in the substances (e.g., milk) that a species (e.g., mammals) should be prepared to consume starting from a very early age. As with many behaviors, metabolic responses to flavors likely reflect a combination of unconditioned and learned responses.

\section{The role of Pavlovian conditioning in appetite regulation}

As a novel flavor is repeatedly paired with calories, such as when an omnivorous animal consumes a novel food, the development of flavor-calorie associations increases the amount of food consumed during an eating bout (i.e., a meal). The initial intent of our research was to document the effect of the development of flavor-calorie associations on general food consumption and weight gain in animals that received a new flavor paired with calories compared to animals just receiving flavors or calories separately. A surprising but reliable result emerged, however, which led us to continue to explore the role of novel flavors that were or were not paired with calories on appetite and body weight over a 3 -week period. The following series of experiments, therefore, examines the effect of novel flavors, consumed in solutions containing either plain water or sugar water, on appetite and weight regulation over a 3-week interval.

We developed a protocol to isolate the learning event (exposure to flavors and/or calories) from its potential effects on appetite and body weight. ${ }^{1}$ A diagram of the treatment is depicted in Fig. 1. Rats were given ad libitum access to lab chow and water for $20 \mathrm{~h}$ each day. During their active period, water and food access were restricted for two 90-min flavorfree and calorie-free windows. Following the first window, rats were given $60 \mathrm{~min}$ of access to a small amount of liquid containing some combination of flavor or no flavor, and calories or no calories. Another 90-min window of no flavor or calories followed the 1-h access, and ended with the return of water and chow until the next treatment $20 \mathrm{~h}$ later. This procedure was repeated daily for 21 days, which should be sufficient time for the development of flavor-calorie associations to have their effects on the dependent measures of interest (appetite and body weight). It should be noted that sucrose solution contains a small but detectable amount of flavor, at least for rats (Rhinehart-Doty, Schumm, Smith, \& Smith, 1994). Nevertheless, adding a salient flavor should greatly enhance the effects of flavor-calorie associations compared to the sugar water condition in which sugar flavor is of low salience. Furthermore, the 2 (flavor vs. no flavor) $\times 2$ (sugar vs. no sugar) design of this experiment allows us to tease apart any effect of the flavor of sugar from its taste.

\section{Experiment 1}

In this experiment, rats received either plain water, sugar water, water with a pomegranate-berry flavor added, and sugar

\footnotetext{
${ }^{1}$ This protocol also mimics a diet-intervention strategy proposed by Roberts $(2004,2006)$ that was motivated by the thesis that learned flavor-calorie associations promote general hunger and overeating. We did not find evidence for this dieting strategy or its putative theoretical mechanisms and thus have omitted major discussion of it from the Introduction and Conclusion.
} 


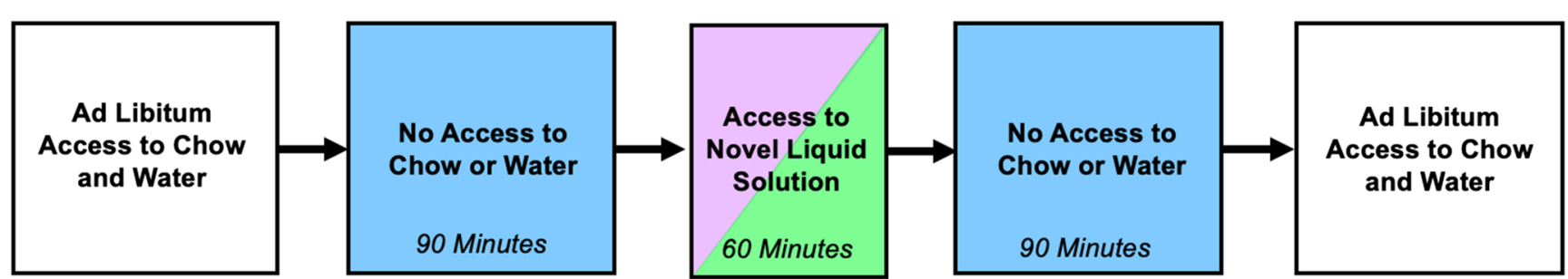

Fig. 1 Schematic of the daily procedural timeline for Experiments 1, 2, and 3

water with the pomegranate-berry flavor added. Rats received their daily access to their proscribed liquid following the protocol described above (Fig. 1) for 21 consecutive days.

\section{Method}

\section{Subjects}

Thirty-two female Long Evans rats (Envigo) were used in this experiment. Subjects were single-housed in transparent plastic tubs with a wood-shaving substrate. Subjects were housed in a vivarium maintained on a reverse 12-h light cycle (lights off at 7 a.m.). Immediately preceding this study, all subjects had participated in a study involving exposure to audiovisual cues, footshock, and a $20 \%$ sucrose solution. During that training, animals were kept at $85 \%$ of their free-feed weight and pair-housed. After that experiment concluded, animals were individually housed and given ad libitum food access for 6 days before the diet intervention procedure of Experiment 1 began. Our decision for reusing these rats reflects ethical considerations with regard to the three Rs of animal research (Fenwick, Griffin, \& Gauthier, 2009), but it is acknowledged as a potential confound of this study, which we discuss further in the conclusion.

\section{Materials}

Mio Liquid Water Enhancer (Kraft Foods, Berry Pomegranate) was used as the novel flavor additive. The solution contains zero calories and is sweetened by a combination of sucralose, acesulfame potassium, and less than $2 \%$ natural flavors. This flavor additive was initially chosen for its lack of calories but is confounded by the fact that it has the properties of a sweet taste. We elaborate on this further in the Discussion and also choose a different flavor additive (peppermint extract) in Experiments 2 and 3. Four solutions were created using separate equipment for the production and dispersion of each to reduce contamination of flavors and calories. Each of the flavored solutions contained 3\% Mio Flavoring, the rest being tap water. Each of the caloric solutions contained $20 \%$ sucrose. Rats were randomly assigned to treatment group. Mean initial body weight per group was $248.12 \pm 2.91 \mathrm{~g}$.

\section{Procedure}

Rats were first individually caged for 6 days and given access to standard lab chow and filtered water ad libitum. This 6-day period allowed subjects to adjust to the novel single-housed environment. Following this 6-day adjustment period, access to food and water was restricted each day from 1 to 5 p.m. Figure 1 displays a complete outline of the daily procedure during this 4-h period. In each condition, a 90-min period elapsed where rats had no access to food or water. When removing food and water from each subject's home cage, care was taken to remove any food hidden within the cage, particularly in the wood shavings. At the conclusion of this 90-min period, each subject received access to a bottle containing $30 \mathrm{ml}$ of their group-assigned liquid solution (water, flavored water, sugar water, or flavored sugar water) for $60 \mathrm{~min}$. At the conclusion of this 60-min access to the bottle, the solution was removed for another $90 \mathrm{~min}$ before having their normal water bottle and chow returned. Although each bottle contained $30 \mathrm{ml}$, the angle at which it was placed in the cage only allowed access to about half of the solution (i.e., $\sim 15 \mathrm{ml}$ ). Rats typically consume around 10 $15 \%$ of their body weight in water (Kuribara et al., 1978 ) in a daily period, and so even if our rats (average starting weight $=248 \mathrm{~g}$ ) consumed all of the liquid, it would only be around half of their daily typical water intake. Body weight measurements and food consumption measurements were made during the first and second 90 -min periods, respectively. This procedure was repeated for 21 consecutive days.

\section{Results and discussion}

Measurements of body weight were taken daily and analyses were conducted using 2-day blocks (Fig. 2a). A mixed ANOVA with a Greenhouse-Geisser correction and with Block as a repeated measure, and Flavor (present or not present) and Sugar (present or not present) as between-subject factors conducted on body weight revealed a main effect of Block, $F(5.197,147.511)=11.821, p<0.001, \eta^{2}=0.019$, indicating that body weight significantly changed over the course of the intervention. This analysis also revealed an interaction between Block and Flavor, $F(5.197,147.511)=$ 5.399, $p<0.001, \eta^{2}=0.009$, but no interaction between 

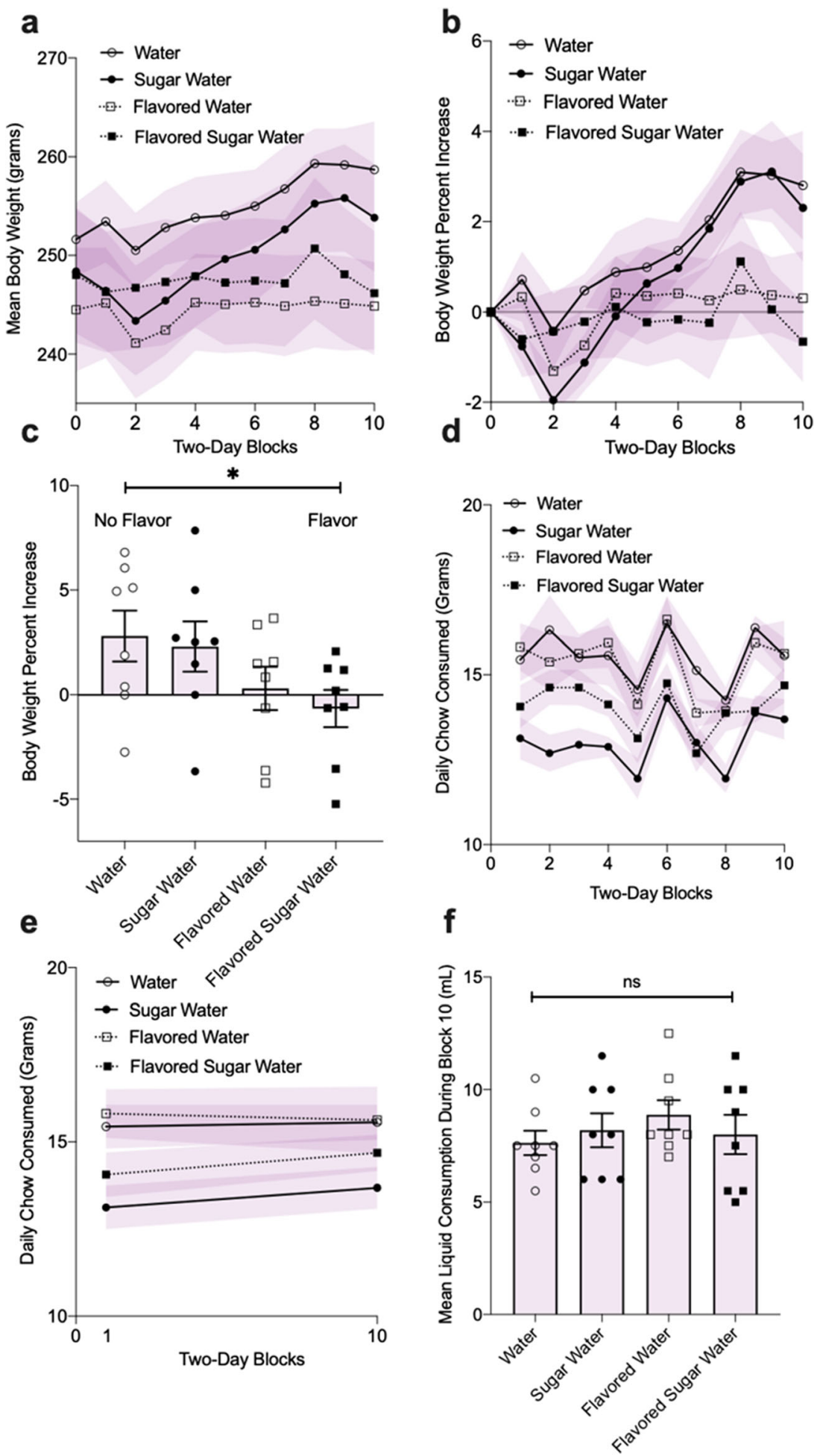

Fig. 2 Data from Experiment 1 using Mio Berry Pomegranate flavoring. Error bars represent SEM. * $p$ value $<.05$, ** $p$ value $<.01$, *** $p$ value $<.001$. (a) Mean body weight (g) following liquid intervention in twoday blocks as a function of each liquid-intervention group. (b) Mean body

weight converted to percent increase. (c) Mean body weight increase on final block of liquid intervention. (d) Daily food consumption in 2-day blocks. (e) Change in daily food consumption from block 1 to block 10 . (f) Mean consumption of the intervention liquid for $1 \mathrm{~h}$ during block 10

Block and Sugar, $F(5.197,147.511)<1.0$, nor was there a three-way interaction, $F(5.197,147.511)<1.0$.

Mean body weights differed between groups at the start of the intervention. Therefore, to better assess the effect of the 
intervention on changes in body weight, we calculated body weight percent change over the course of 3 weeks (Fig. 2b). A mixed ANOVA with a Greenhouse-Geisser correction and with Block as a repeated measure, and Flavor (present or not present) and Sugar (present or not present) as between-subject factors conducted on body weight percent increase revealed a main effect of Block, $F(5.433,152.130)=14.465, p<0.001$, $\eta^{2}=0.105$, an interaction between Block and Flavor, $F(5.433,152.130)=6.069, p<0.001, \eta^{2}=0.044$, but no interaction between Block and Sugar, $F(5.433,152.130)<1.0$, nor was there a three-way interaction, $F(5.433,152.130)=$ $1.186, p=0.318, \eta^{2}=0.009$.

Due to these interactions, and because we were more interested in the final percentage of weight gain or loss from the intervention, a 2 (flavor vs. no flavor) $\times 2$ (sugar vs. no sugar) ANOVA conducted on body weight percent change in the tenth block (i.e., the last 2 days, Fig. 2c) of the intervention procedure revealed a main effect of Flavor, $F(1,28)=6.260, p$ $=0.018, \eta^{2}=0.180$. No effect of Sugar was found, $F(1,28)<$ 1.0 , nor was there a Flavor x Sugar interaction on weight gain, $F(1,28)<1.0$. Thus, when the liquid solution contained Mio flavoring, natural weight gain associated with the removal of restricted feeding was attenuated. Interestingly, the presence of sugar in the flavored liquid solutions did not affect this attenuation in weight gain, $F<1.0$, nor did it cause increased weight gain for the sugar water condition compared to the water condition, $F<1.0$.

Measurements of daily food consumption were also taken. Following the daily intervention procedure, rats were always given access to $85 \mathrm{~g}$ of Purina Lab Chow. The following day, we weighed the amount of remaining food after we had removed it from the animal's cage and subtracted that value from 85 to compute daily consumption. Food consumption was also analyzed in 2-day blocks (Fig. 2d). While the development of flavor-calorie associations has been shown to increase consumption of food containing that specific flavor, the effects of these associations on general food consumption is unclear. Roberts (2004) theorized that learning flavor-calorie associations should increase general appetite after detection of the flavor, because it serves as a signal that high quality food is available and should be consumed, but this has yet to be adequately tested. Nevertheless, because we observed differences in weight gain, we were not only interested in how the amount of chow consumed differed between groups, but also how the consumption differed within condition over time due to the respective treatments. A mixed ANOVA was conducted on food consumption data with two between-subject factors (Sugar and Flavor) and one within-subject factor (Blocks 1 and 10; Fig. 2e). A main effect of Sugar was found, $F(1,28)$ $=10.352, p=0.003, \eta^{2}=0.258$, such that rats that drank liquid solutions containing sugar consumed less food than rats that drank liquid solutions without sugar. This could be explained by the fact that the animals were receiving additional calories from their sugar in their liquid intervention solution. There was no main effect of Flavor, $F(1,28)=1.235, p=0.276, \eta^{2}$ $=0.031$, nor an interaction between Flavor and Sugar, $F(1,28)$ $<1.0$. We also did not see any evidence that learning a flavorcalorie association affected food consumption. No main effect of Block ( 1 vs. 10 ) was found, $F(1,28)<1.0$, nor was there an interaction between Block and Sugar, $F(1,28)<1.0$, or Block and Flavor, $F(1,28)<1.0$, nor a three-way interaction between Block x Sugar x Flavor, $F(1,28)<1.0$. Daily chow consumed, therefore, does not appear to explain the group differences in weight gain. Critically, though the animals that drank flavored solutions did not gain weight as much as did animals that drank non-flavored solutions, these animals did not eat any less food than the animals who gained weight.

Is it possible that these differences in weight gain could reflect differences in the amount of liquid consumed (e.g., overconsumption or no consumption) during the 1-h intervention period? We believe this to be unlikely given the small amount of liquid $(\sim 15 \mathrm{ml})$ that was given in a relatively short period of time. To test this, we took pre- and post-bottle weight measurements on the last 2 days of the intervention procedure for all rats. These data are displayed in Fig. $2 \mathrm{f}$, and a one-way ANOVA revealed no effect of condition on amount of liquid consumed during the tenth block of intervention, $F(3,28)<1.0$. Interestingly, while rats drank more of the sugar water compared to the unsweetened water, this difference was not as large as one might expect given rats typically prefer sweetened water. This is possibly the result of rats having previous exposure to the sucrose solution but could also be due to the small amount of liquid that was provided to already thirsty rats. Further, that all rats consumed about the same amount of liquid during the intervention period suggests the observed patterns in weight gain are due to contents of the liquid solution and not how much of it was consumed.

The animals that drank the flavored solutions did not gain weight, yet they also did not consume any less food than the non-flavor controls. This suggests there may have been differences in metabolic responses evoked by the flavored solutions. Specifically, the $3 \%$ Mio flavored solutions, which were sweet and flavorful, may have evoked a large metabolic response from the rats. For the flavored water condition, no calories followed this flavor exposure, and so metabolism could only operate on the body's existing energy stores (e.g., glycogen and lipids). Similarly, given the strong sweetness and flavor of the Mio-flavored sugar solution, the magnitude of the metabolic response may have exceeded the appropriate response magnitude for that amount of incoming calories. In the absence of a direct measure of metabolic response, this interpretation is speculative. This interpretation is also similar in principle to research that demonstrates the potential downsides of artificial sweeteners from a learning perspective (Davidson \& Swithers, 2004; Swithers, 2013; Swithers \& Davidson, 2008). Through experience, or perhaps evolved 
predispositions, sweetness is a strong signal of incoming calories. Frequent consumption of artificially sweetened foods, therefore, can result in sweetness becoming an unreliable signal of incoming calories, thereby lowering the magnitude of metabolic responding to sweet tastes. In turn, when one actually consumes a high-sweet, high-calorie food item, the metabolic response is insufficient to metabolizing the calories and the excess is stored, potentially resulting in weight gain. Thus, in our experiment, an unconditioned or learned association between sweetness and calories may have resulted in the Mio-flavored solutions eliciting a large metabolic response for either no incoming calories (Flavored Water) or fewer incoming calories than was expected given the level of sweetness (Flavored Sugar Solution). While the Mio flavoring was entirely novel to the subjects at the start of the experiment, all rats had prior experience with a sweetened liquid solution that contained calories (20\% sucrose water). This creates some difficulty in drawing inferences about the extent to which the metabolic response to the Mio flavoring was due to some sort of unconditioned metabolic response to flavors or to generalization because the rats had learned that other sweet solutions contain calories. Additionally, that Mio contains acesulfame potassium is also troubling because this substance has been shown to increase consumption and weight gain under some conditions (Swithers, Baker, \& Davidson, 2009). To obviate these confounding factors, in Experiment 2, we replicated the procedure of Experiment 1 using a peppermint extract as the flavor additive. The peppermint extract on its own was not sweet.

\section{Experiment 2}

The surprising result from Experiment 1 was the suppressing effect on weight gain by consuming an initially novel flavor, despite no change in daily amount of food consumed. These unexpected findings raise the hypothesis that novel flavors might unconditionally elicit metabolic responses, even if those novel flavors exist in liquid solutions that contain no calories. To investigate this hypothesis and rule out any effect of the sweetness of the novel flavor used in Experiment 1, we replicated the procedure of Experiment 1 with new rats, and used peppermint extract as the novel flavoring agent. Critically, none of the animals had prior experience with the peppermint flavoring, and this flavor was highly distinct from any other flavors the animals had previously experienced.

\section{Method}

\section{Subjects}

Thirty-two female Long Evans rats (Envigo) were used in this experiment. Rats had similar prior experience with cues, footshock, sucrose solution, and food restriction as did rats in Experiment 1. Housing and acclimation were as described for Experiment 1.

\section{Materials}

The materials used in Experiment 2 were nearly identical to those used in Experiment 1, with the exception being that flavored solutions were flavored with $0.039 \%$ McCormick peppermint extract. Rats were pseudorandomly assigned to group and care was taken to ensure there were minimal initial differences in mean body weight between the four groups (all groups mean initial body weight was $254.5 \pm 0.76 \mathrm{~g}$ ).

\section{Procedure}

The procedure was identical to that used in Experiment 1 (Fig. 1).

\section{Results and discussion}

Mean body weight over the 3-week period is displayed in Fig. 3a. A mixed ANOVA with a Greenhouse-Geisser correction with Block as the repeated measure and Flavor (present or not present) and Sugar (present or not present) as between-subject factors conducted on body weight revealed a main effect of Block, $F(4.390,122.920)=20.210, p<0.001, \eta^{2}=0.017$, indicating that body weight significantly changed over the course of the intervention. There was a significant interaction between Block and Flavor, $F(4.390,122.920)=$ 2.525, $p=0.006, \eta^{2}=0.002$, but no interaction between Block and Sugar, $F(4.390,122.920)=1.133, p=0.356, \eta^{2}$ $=0.001$, nor a three-way interaction, $F(4.390,122.920)<$ 1.0. We then performed similar analyses after converting body weight to body weight percent increase (Fig. 3b) and found a main effect of Block, $F(4.682,131.096)=18.079$, $p<0.001, \eta^{2}=0.119$, but no interactions between Block and Flavor, $F(4.682,131.096)=1.811, p=0.120, \eta^{2}=$ 0.012 , between Block and Sugar, $F(4.682,131.096)=$ $1.182, p=0.322, \eta^{2}=0.008$, nor a three-way interaction, $F(4.682,131.096)<1.0$.

We were most interested in the final effect of the intervention on body weight. A two (flavor vs. no flavor) $\times$ two (sugar vs. no sugar) ANOVA conducted on the body weight data from the tenth block (i.e., last 2 days) of the intervention (Fig. 3c) revealed a main effect of Flavor, $F(1,28)=10.273, p=0.003, \eta^{2}=0.242$. There was no effect of Sugar, $F(1,28)=2.339, p=0.137, \eta^{2}=0.055$, nor was there an interaction between Flavor and Sugar, $F(1,28)=1.866, p=0.183, \eta^{2}=0.044$. While flavor and sugar did not interact, the flavored water condition appeared to gain less weight than the flavored sugar water 

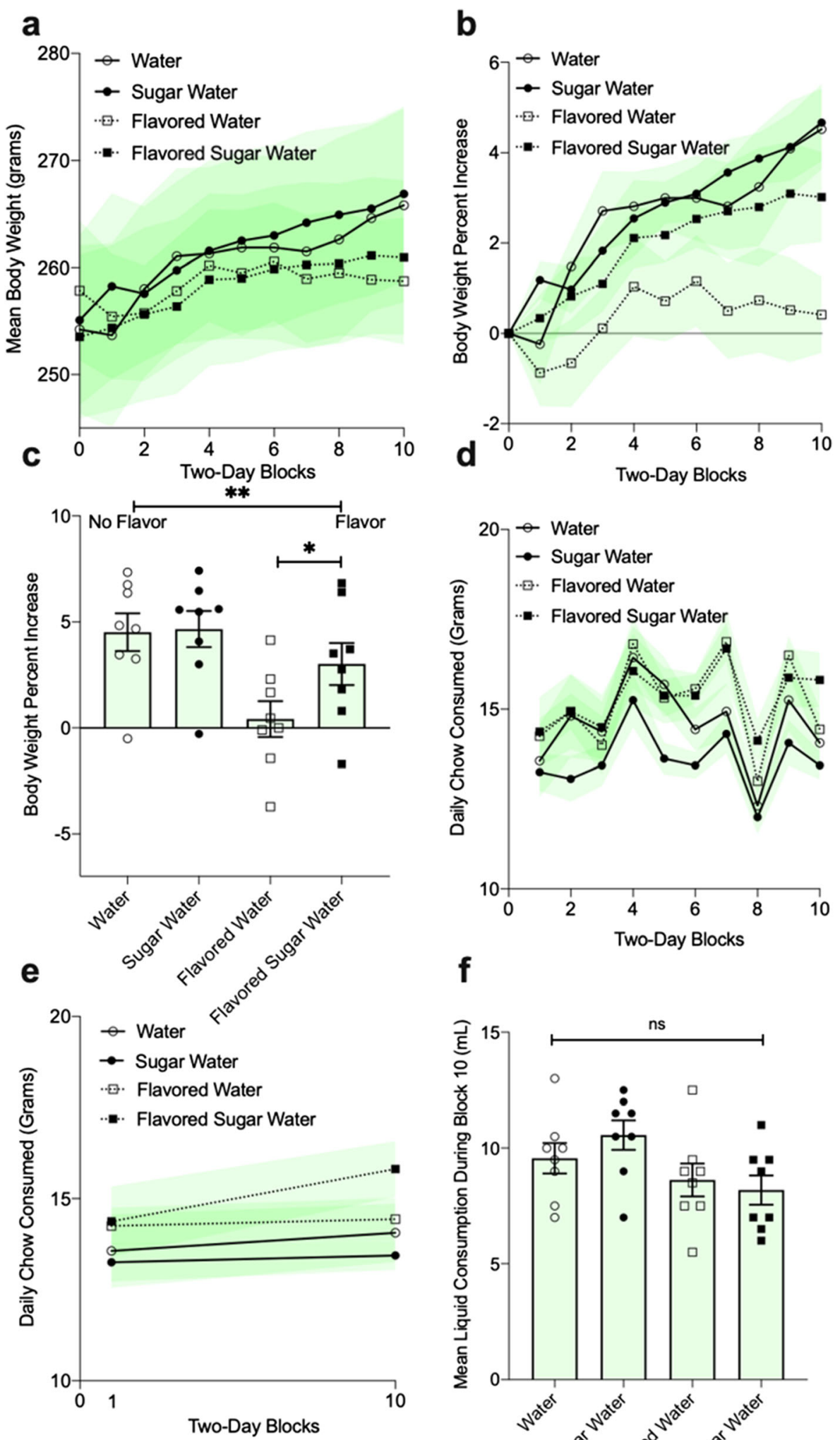

f

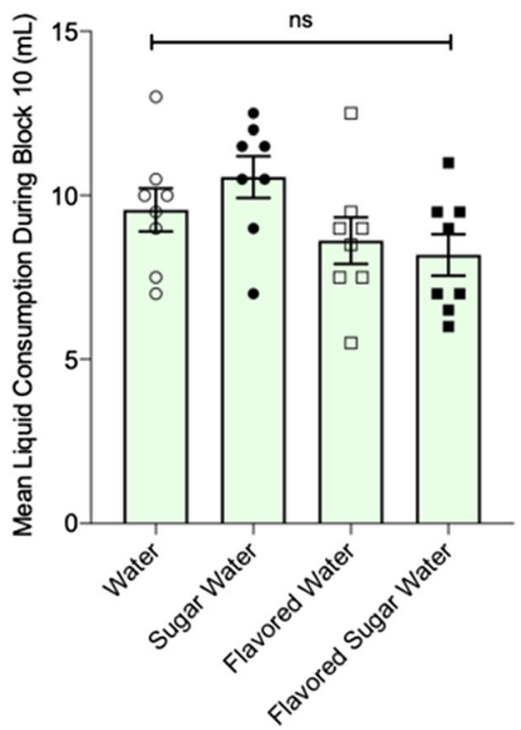

Fig. 3 Data from Experiment 2 using McCormick peppermint extract as flavoring. Error bars represent SEM. * $p$ value $<.05$, ** $p$ value $<.01$, *** $p$ value $<.001$. (a) Mean body weight (g) following liquid intervention in 2day blocks as a function of each liquid-intervention group. (b) Mean body

weight converted to percent increase. (c) Mean body weight increase on final block of liquid intervention. (d) Daily food consumption in 2-day blocks. (e) Change in daily food consumption from block 1 to block 10. (f) Mean consumption of the intervention liquid for $1 \mathrm{~h}$ during block 10 
condition. Indeed a simple effect of sugar was found for flavored liquids, $F(1)=4.191, p=0.050$. These results replicate what was found in Experiment 1, that repeated consumption of a novel flavored liquid solution during a window of no access to other flavors or calories attenuated weight gain. Unlike in Experiment 1, this effect in Experiment 2 appears to be specific to the flavored water solution, because it was not found in rats consuming the flavored sugar solution (but note the lack of significant interaction between flavor x sugar). Additionally, this replicated effect was achieved using peppermint as a flavor additive, which is quite different to the Pomegranate Mio flavor solution used in Experiment 1, thereby demonstrating the generality of the effect of novel flavors.

A summary of daily food consumption as a function of intervention condition is displayed in Fig. 3d. A mixed ANOVA was conducted with two between-subject factors (Sugar and Flavor) and Block (1 and 10) as a repeated measure to examine trends in daily food consumption over the 3-week period (Fig. 3e). Unlike in Experiment 1 , there was no main effect of Sugar, $F(1,28)<1.0$, though the sugar water condition did again nominally consume the least amount of food. There was a marginal effect of Flavor, $F(1,28)=3.519, p=0.071, \eta^{2}=0.108$, such that rats that drank flavored beverages ate slightly more food. There was no significant interaction between Sugar and Flavor, $F(1,28)=1.004, p=0.325, \eta^{2}=0.031$. We also failed to observe any change in eating as a result of learning, as there was no difference in consumption between the first and last Block, $F(1,28)=2.168, p=$ $0.152, \eta^{2}=0.020$, and there was no interaction between Block and Sugar, $F(1,28)<1.0$, Block and Flavor, $F(1,28)<1.0$, and no three-way interaction between Block x Sugar x Flavor, $F(1,28)<1.0$. While the flavored sugar water group increased daily food consumption over training, this difference was not significant, $t(7)=1.475$, $p=0.184$. We again measured the amount of liquid consumed during the 1-h intervention period on the last 2 days of the intervention and did not find differences between the conditions, $F(3,28)=2.55, p=0.076, \eta^{2}=$ 0.215 (Fig. 3f).

The findings from Experiment 2 strengthen our interpretation of the data in Experiment 1, that consuming a novel flavor results in the elicitation of metabolic responses that then metabolize existing stored energy in the absence of incoming calories. This is one plausible explanation of the main effect of flavor in both experiments despite no differences in daily food consumption. It might also explain why the presence of sugar did not affect weight gain in Experiment 1 but did in Experiment 2. That is, the sugar solution flavored with
Mio may have evoked a metabolic response much larger than was needed for the calories within that solution. In Experiment 2, the potentially less salient and certainly more novel peppermint flavor may have elicited a slightly smaller metabolic response, and so when the flavored solution also contained calories those calories were less readily metabolized by the evoked responses. If this interpretation of the data is correct, it would suggest that metabolic responses can be elicited by entirely novel flavors, even prior to learning about its post-ingestive consequences. While learning processes can certainly affect the magnitude and identity of these responses, it would also make sense for organisms to have, a priori, a baseline metabolic response to novel flavors, to maximize nutrient absorption and due to the potentially lethal consequences that could follow ingesting a novel food. To investigate the existence of these potential unconditioned metabolic responses to novel flavors, we reasoned that varying the intensity of the flavor of the liquid intervention may affect the intensity of the evoked metabolic response.

\section{Experiment 3}

A viable interpretation of the data from Experiments 1 and 2 is that unconditioned metabolic responses are elicited by the consumption of a flavor, even in the absence of learned caloric consequences of that flavor. Here we test whether varying the intensity of the flavor will vary the intensity of its unconditioned effects of suppression of weight gain in free-feeding rats. We repeated the wateronly and peppermint-water group treatments from Experiment 2, but for the peppermint-water manipulation we gave rats access to a solution containing a weak flavor, a medium flavor, or a strong flavor.

\section{Method}

\section{Subjects}

Thirty-two female Long Evans rats (Envigo) were used in this experiment. Rats had similar prior experience with cues, footshock, sucrose solution, and food restriction as did rats in Experiment 1. Housing and acclimation were as described for Experiment 1.

\section{Materials}

McCormick peppermint extract was again used as the flavoring agent. The medium flavor condition was given 
a solution containing $0.039 \%$ flavoring, which is the same amount of flavor as used in the flavor-water condition from Experiment 2. Rats in the strong condition were given twice that concentration of flavoring $(0.077 \%)$ and the weak condition was given half the concentration of flavoring as the medium condition $(0.019 \%)$. Rats were pseudo-randomly assigned to each condition and care was taken to ensure there were minimal initial differences in mean body weight between the four groups (all groups mean initial body weight was $259.1 \pm 0.79 \mathrm{~g}$ ).

\section{Procedure}

The procedure was identical to that used in Experiment 2 (Fig. 1). After 21 days of daily liquid intervention, rats in the medium flavor condition and water condition were given a preference test for the medium flavored solution or water. Each rat $(n s=16)$ received simultaneous access to both solutions and the time spent drinking from each bottle was recorded for $5 \mathrm{~min}$. The amount of liquid consumed from each bottle after $1 \mathrm{~h}$ was measured by subtracting pre- and post-bottle weights.

\section{Results and discussion}

Mean body weight over the 3-week period is displayed in Fig. 4a. A mixed ANOVA with a Greenhouse-Geisser correction conducted on body weight data with Block as a repeated measure and Group as a between-subject factor revealed a main effect of Block, $F(3.651,102.242)=$ $12.522, p<0.001, \eta^{2}=0.017$, indicating that body weight significantly changed over the course of our intervention. There was also a significant interaction between Block and Group, $F(3.651,102.242)=2.715, p=0.004, \eta^{2}=$ 0.011 . After converting body weight to body weight percent increase (Fig. 4b), we conducted identical analyses and found a main effect of Block, $F(3.566,528.4)=$ 7.518, $p<0.001, \eta^{2}=0.080$, and an interaction between Block and Group, $F(3.566,528.4)=2.686, p=0.005, \eta^{2}$ $=0.086$. We were most interested in the overall effect of the intervention on weight gain, and so we conducted a one- (Group) way ANOVA on the tenth block of body weight (Fig. 4c), which revealed a main effect of Group, $F(3,28)=6.713, p=0.001, \eta^{2}=0.418$. While we did not observe a strong dose-dependent response function, all groups with a flavored liquid solution showed attenuation of weight gain compared to the water group. Tukey HSD post hoc analyses support these observations. Compared to the water group, rats gained less weight in Group Strong flavor, $t=3.503, p=0.008$, Cohen's $d=1.763$, Group Medium flavor, $t=4.171, p=0.001$, Cohen's $d=$
2.163, and Group Weak flavor, $t=2.763, p=0.047$, Cohen's $d=1.353$. These results suggest an apparent role of the peppermint flavoring in reducing weight gain.

A mixed ANOVA with one between-subject factor (Group) and Block (1 and 10) as a repeated-measure conducted on food consumption revealed no effect of Block, $F(1,28)=$ 2.157, $p=0.153, \eta^{2}=0.068$, and critically, no effect of Group, $F(3,28)<1.0$ (Figs. $4 \mathrm{~d}$ and $4 \mathrm{e}$ ). There was also no interaction between Block and Group, $F(3,28)<1.0$. The differences in body weight gain despite the lack of differences in food consumption suggests evoked metabolic responses by the consumption of a novel flavor, despite it not signaling a caloric outcome (US).

An alternative explanation of these data could be that the rats found the peppermint-flavored liquid solutions aversive, and that the lack of liquid consumption during the 60-min exposure interval was responsible for the lack of weight gain. We had anecdotal reasons to doubt this, because we often observed the rats eagerly approach the liquid solutions, presumably because they were thirsty following $90 \mathrm{~min}$ of no access to food or water. Additionally, in Experiments 1 and 2, we found no group differences in the amount of liquid consumed during the 1-h intervention period on the tenth block. Nevertheless, to empirically demonstrate with a different procedure that the rats did not find the peppermint-flavored water aversive, we restricted rats from food and water for $120 \mathrm{~min}$, and then gave the rats in Group Medium flavor simultaneous access to the medium flavored Peppermint solution and a water solution after completing 21 days of access to the medium flavored Peppermint solution. We measured the amount of time spent drinking from each bottle during a 5-min observation period, and the pre- and postbottle weights following 1-h access to both bottles. A paired samples t-test revealed no difference in the amount of time spent at each bottle during $5 \mathrm{~min}$ of observation, $t(7)<1.0$. In addition, there was no difference in the amount of liquid solution consumed from each bottle following an hour's exposure with simultaneous access, $t(7)<1.0$. We also ran an identical procedure for rats in the water condition, thus allowing them access to the medium-flavored solution for the very first time, along with access to water. Again, we saw no difference in amount of time spent drinking from either bottle, $t(7)<1.0$, nor was there a difference in the amount of liquid consumed after 60 -min access, $t(7)<$ 1.0. These data support our anecdotal experience of the rats willingly consuming the flavored solution during the 60 -min intervention period, and rules out dehydration or overhydration as explanations for the lack of weight gain observed in the flavored solution conditions. It also 


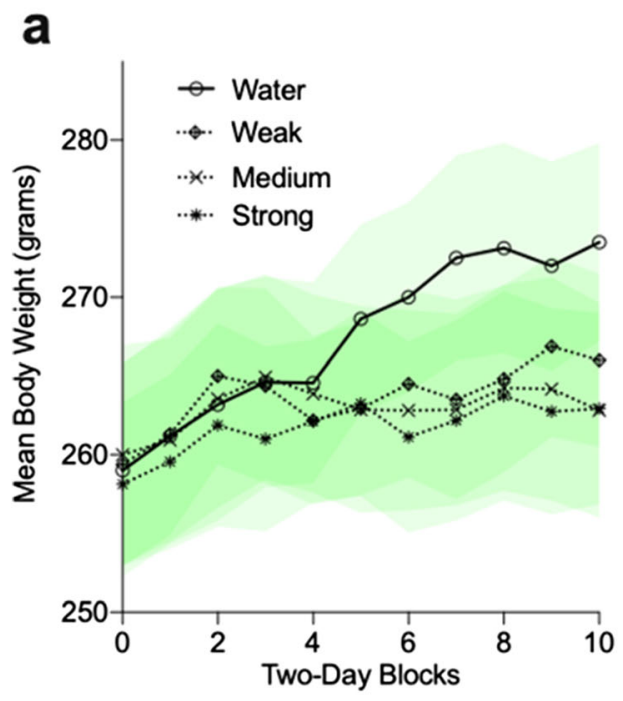

b
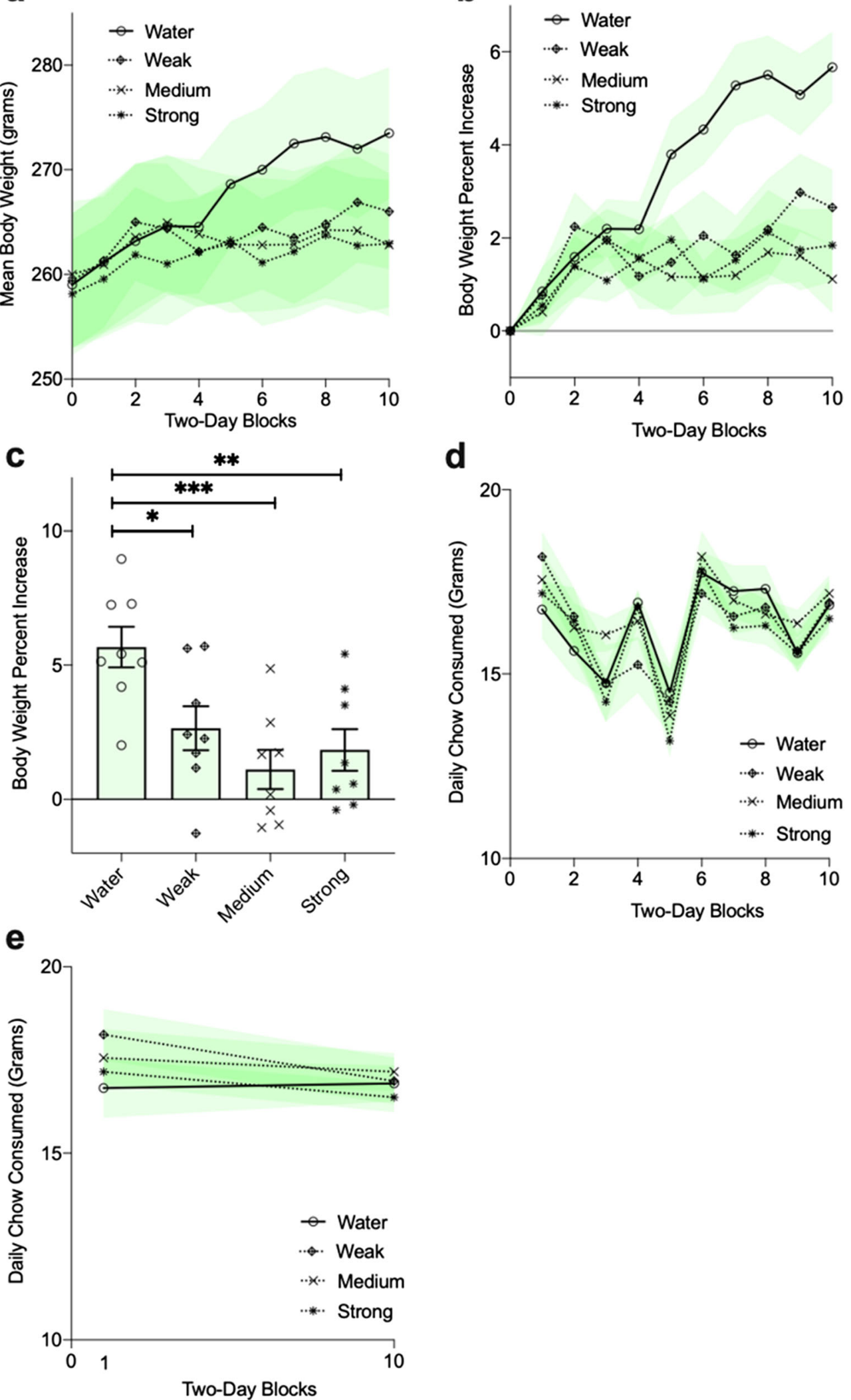

Fig. 4 Data from Experiment 3 using McCormick peppermint extract as flavoring. Error bars represent SEM. $* p$ value $<.05$, ** $p$ value $<.01$, $* * * p$ value $<.001$. (a) Mean body weight (g) following liquid intervention in 2-day blocks as a function of each liquid-intervention group. (b)

Mean body weight converted to percent increase. (c) Mean body weight increase on final block of liquid intervention. (d) Daily food consumption in 2-day blocks. (e) Change in daily food consumption from block 1 to block 10 
shows that the Peppermint flavor wasn't unconditionally aversive as rats that had previously had access only to water showed no avoidance to the Peppermint solution the first time they were given access.

\section{Conclusions}

Across three experiments, we report consistent evidence that the consumption of flavored liquids in between calorie-free and flavor-free windows can attenuate weight gain. Because there were no differences in the amount of daily food consumed, we speculate that these data can be interpreted as evidence for unconditioned metabolic responses to consuming a flavor. We found no evidence that an acquired flavor-calorie association in the flavored sugar water group resulted in any change in body weight or daily food consumption of normal chow, which does not support Roberts' (2004) theory. The effect of an initially novel flavor was replicated three times and with two flavors (Berry Pomegranate and Peppermint). The lack of an effect of flavor-calorie associations on body weight and food consumption was replicated twice, once for each type of flavor.

Finally, the lack of any discernable preference for or avoidance of the flavored water compared to plain water suggests that the suppressing effects of flavor water exposure on body weight cannot be explained by rats preferring or avoiding either water or the peppermint-flavored solutions.

Taken together, these data suggest that the ingestion of unpaired flavors can unconditionally suppress weight gain, at least under the strict parameters of our experimental design (see Fig. 1). We believe this can be explained by unconditioned metabolic responses that are elicited by the consumption of flavors. While learning processes have been demonstratively shown to influence the magnitude and identity of metabolic responses, the existence of an unconditioned, reflexive metabolic response to novel-flavor consumption in the absence of calories is likely adaptive given the paradox of feeding (Woods, 1991). The paradox of feeding is that while feeding is a necessary behavior for survival, consuming food threatens some aspects of homeostasis by introducing exogeneous substances into the body, some of which can be harmful. Even vital nutrients are only physiologically tolerable within a restricted range. Thus, post-ingestive metabolic and other physiological responses are critical to an organism's survival and are unlikely to be entirely dictated by learning processes because relying solely on learned responses could have fatal consequences. An analogy can be drawn to the emergency self-braking feature in many modern cars. When faced with an incoming object, the driver can choose to apply the brake at an appropriate level based on his/her experience. Nevertheless, even if the brake is not pressed, the emergency self-braking system will stop the car, though not always in a well calibrated or smooth manner. Thus, well-calibrated metabolic responses to flavors may dominate when the organism has learned about the post-ingestive consequences of a flavor, but it would be problematic for an organism not to have any metabolic response to the detection of a flavor, because a whole host of post-ingestive consequences could potentially follow. This potential unconditioned metabolic response to flavors may also reflect some sort of prepared learning, as it is likely that the vast majority of flavors ever detected by an organism and its ancestors were followed by a caloric outcome (Seligman, 1970). That said, to our knowledge, little evidence exists to accurately assess the possibility of unconditioned metabolic responses to flavors.

One limitation to our interpretation is that we are indirectly inferring metabolic responses due to observed differences in weight gain in the absence of differences in food consumption. This was the result of our initiating these experiments for separate reasons, but then continuing to interrogate the emerging behavioral patterns. A more direct way to measure unconditioned metabolic responses to a novel or familiar flavor would be to directly measure a metabolic response following exposure to a novel flavor that does not contain calories. Ideally, these measurements would occur during both the first exposure to the flavor and after 21 days of daily exposure to it, and would be compared to those same measurements but to a flavor that is paired with calories. There are various measurements that more directly target the potential metabolic response(s) responsible for this effect, including insulin release, thermoregulation, and energy expenditure. None of these targets work in isolation and may be individually or collectively responsible for this effect, though measuring metabolic response is notoriously challenging (Speakman, 2013). Supportive of our interpretation, Dhillon, Lee, and Mattes (2017) measured Cephalic Phase Insulin Responses to both sucrose (a caloric sweetener) and sucralose (an artificial sweetener with minimal calories) in humans and found no differences in the magnitude of insulin response to liquid solutions that contained either of these sweeteners, even after a 2-week period of repeated daily exposure to those liquids. While the Dhillon et al. procedure utilizes a sweet taste, it may be that our non-caloric, and in Experiments 2 and 3 non-sweet, flavors also elicited a metabolic response that failed to habituate after a 3-week period. It is possible that with more time, these responses may eventually habituate, but their apparent lack of habituation in our 3-week procedure may reflect the strength of these responses and/or their evolutionary significance (i.e., resistance to habituation).

An additional limitation of these studies is our use of rats that had previous experimental experience with a small amount of sugar water. Our decision to re-use rats reflects commitments to, as outlined by the 3 Rs (Fenwick et al., 2009), reusing animals when possible in experimental animal research. This may have had some influence on liquid consumption because in Experiments 1 and 2 we did not observe a sizeable difference 
in drinking sugar compared to regular water. However, this may be due to other reasons like the short period of time rats had with these solutions as well as the small amount of liquid actually given. Further, the lack of differences in liquid consumption support our view that it was critically the contents of the liquid solution that influenced weight gain or lack thereof, and not the amount consumed. Finally, we did not measure water consumption in the ad libitum period, which may potentially explain or illuminate the observed effects.

A tempting conclusion of these experiments may be that they support the use of diet sodas or other flavored but caloriefree beverages (e.g., peppermint tea or other floral teas) as part of a weight-loss strategy. We caution against this interpretation, primarily due to a number of studies suggesting that regular consumption of artificial sweeteners renders sweetness an unreliable predictor of calories, resulting in an inadequate metabolic response following consuming foods that are sweet and also calorically dense (Swithers, 2013; Swithers \& Davidson, 2008; Swithers, Martin, \& Davidson, 2010, but see Rogers et al., 2016, for an important discussion on human vs. animal models). Additionally, our results were obtained by having animals consume the non-caloric flavor in the middle of an extended flavor- and calorie-free windows, which is not often how artificially sweetened beverages are consumed (i.e., as a part of a larger meal). Extended use of this method might also be susceptible to habituation, which was not observed in our 3-week intervention period.

While the reported studies were initially motivated by the literature on Pavlovian learning and appetite (Myers, 2018; Roberts, 2004, 2006; Sclafani, 2018), we failed to find an effect of learning a Pavlovian relationship between flavor and calories on weight gain or daily food consumption. Instead, we appear to have discovered that novel flavors can unconditionally suppress weight gain, which we speculate may be caused by metabolic responses that are elicited by entirely novel (at first introduction) flavor stimuli, despite those stimuli not containing calories. While it may be possible to conceive dietingintervention strategies based on these results, more proximally, this knowledge further demonstrates an interaction between unconditioned and learned responses that guide animals to successfully navigate their environments.

Open Science Statement The data for Experiments 1, 2, and 3 can be found at https://osf.io/9vazy/?view_only= bd41698c12a94f91bf45a8b503fe5794. The study was not preregistered.

\section{References}

Apovian, C. M. (2016). Obesity: definition, comorbidities, causes, and burden. The American Journal of Managed Care, 22(7 Suppl), s176-85. Retrieved from http://www.ncbi.nlm.nih.gov/pubmed/ 27356115

Bartoshuk, L. M., \& Beauchamp, G. K. (1994). Chemical Senses. Annual Review of Psychology, 45(1), 419-449. https://doi.org/10.1146/ annurev.ps.45.020194.002223

Birch, L L, McPhee, L., Steinberg, L., \& Sullivan, S. (1990). Conditioned flavor preferences in young children. Physiology \& Behavior, 47(3), 501-505. Retrieved from http://www.ncbi.nlm.nih.gov/pubmed/ 2359760

Birch, Leann L. (1999). Development of Food Preferences. Annual Review of Nutrition, 19(1), 41-62. https://doi.org/10.1146/annurev. nutr.19.1.41

Bolles, R. C., Hayward, L., \& Crandall, C. (1981). Conditioned taste preferences based on caloric density. Journal of Experimental Psychology. Animal Behavior Processes, 7(1), 59-69. Retrieved from http://www.ncbi.nlm.nih.gov/pubmed/7229574

Brunstrom, J. M., \& Mitchell, G. L. (2007). Flavor-nutrient learning in restrained and unrestrained eaters. Physiology \& Behavior, 90(1), 133-141. https://doi.org/10.1016/j.physbeh.2006.09.016

Capaldi, E. D. (1996). Conditioned food preferences. In Why we eat what we eat: The psychology of eating. (pp. 53-80). https://doi.org/10. 1037/10291-003

Capaldi, E. D., Hunter, M. J., \& Lyn, S. A. (1997). Conditioning with taste as the CS in conditioned flavor preference learning. Animal Learning \& Behavior, 25(4), 427-436. https://doi.org/10.3758/BF03209849

Davidson, T. L., \& Swithers, S. E. (2004). A Pavlovian approach to the problem of obesity. International Journal of Obesity, 28(7), 933935. https://doi.org/10.1038/sj.ijo.0802660

Dhillon, J., Lee, J. Y., \& Mattes, R. D. (2017). The cephalic phase insulin response to nutritive and low-calorie sweeteners in solid and beverage form. Physiology \& Behavior, 181, 100-109. https://doi.org/10. 1016/J.PHYSBEH.2017.09.009

Fenwick, N., Griffin, G., \& Gauthier, C. (2009). The welfare of animals used in science: how the \&quot;Three Rs\&quot; ethic guides improvements. The Canadian Veterinary Journal $=$ La Revue Veterinaire Canadienne, 50(5), 523-530. Retrieved from http:// www.ncbi.nlm.nih.gov/pubmed/19436640

Kuribara, H., Hayashi, T., Alam, M. R., Tadokoro, S., \& Miura, T. (1978). Automatic measurement of drinking in rats: Effects of hypophysectomy. Pharmacology Biochemistry and Behavior, 9(5), 697-702. https://doi.org/10.1016/0091-3057(78)90224-1

Mehiel, R., \& Bolles, R. C. (1984). Learned flavor preferences based on caloric outcome. Animal Learning \& Behavior, 12(4), 421-427. https://doi.org/10.3758/BF03199989

Myers, K P, \& Hall, W. G. (1998). Evidence that oral and nutrient reinforcers differentially condition appetitive and consummatory responses to flavors. Physiology \& Behavior, 64(4), 493-500. https://doi.org/10.1016/s0031-9384(98)00106-1

Myers, K. P. (2018). The convergence of psychology and neurobiology in flavor-nutrient learning. Appetite, 122, 36-43. https://doi.org/10. 1016/j.appet.2017.03.048

Myers, K.P., \& Sclafani, A. (2006). Development of learned flavor preferences. Developmental Psychobiology, 48(5), 380-388. https://doi. org/10.1002/dev.20147

Pavlov, I. P. (1927). Conditioned reflexes: An investigation of the physiological activity of the cerebral cortex. https://doi.org/10.5214/ans. 0972-7531.1017309

Pavlov, I. P. (1910). The work of the digestive glands (2nd. English Edition). Retrieved from https://archive.org/details/ workofdigestiveg00pavlrich/page/n17

Rhinehart-Doty, J. A., Schumm, J., Smith, J. C., \& Smith, G. P. (1994). A non-taste cue of sucrose in short-term taste tests in rats. Chemical Senses, 19(5), 425-431. https://doi.org/10.1093/chemse/19.5.425

Roberts, S. (2004). Self-experimentation as a source of new ideas: Ten examples about sleep, mood, health, and weight. Behavioral and Brain Sciences, 27(02). https://doi.org/10.1017/s0140525x04000068 
Roberts, S. (2006). The Shangri-La diet: The no hunger eat anything weight-loss plan. Penguin.

Rogers, P. J., Hogenkamp, P. S., De Graaf, C., Higgs, S., Lluch, A., Ness, A. R., ... Mela, D. J. (2016). Does low-energy sweetener consumption affect energy intake and body weight? A systematic review, including meta-analyses, of the evidence from human and animal studies. International Journal of Obesity, Vol. 40, pp. 381-394. https://doi.org/10.1038/ijo.2015.177

Sclafani, A. (2001). Psychobiology of food preferences. International Journal of Obesity, 25(S5), S13-S16. https://doi.org/10.1038/sj.ijo. 0801905

Sclafani, A. (2018). From appetite setpoint to appetition: 50 years of ingestive behavior research. Physiology \& Behavior, 192, 210 217. https://doi.org/10.1016/J.PHYSBEH.2018.01.001

Seligman, M. E. (1970). On the generality of the laws of learning. Psychological Review, 77(5), 406-418. https://doi.org/10.1037/ h0029790

Small, D. M., \& Prescott, J. (2005). Odor/taste integration and the perception of flavor. Experimental Brain Research, 166(3-4), 345-357. https://doi.org/10.1007/s00221-005-2376-9

Smith, G. P. (1995). Pavlov and appetite. Integrative Physiological and Behavioral Science, 30(2), 169-174. https://doi.org/10.1007/ BF02691685

Smotherman, W. P., \& Robinson, S. R. (1987). Prenatal expression of species-typical action patterns in the rat fetus (Rattus norvegicus). Journal of Comparative Psychology, 101(2), 190-196. https://doi. org/10.1037/0735-7036.101.2.190

Speakman, J. R. (2013). Measuring energy metabolism in the mouse theoretical, practical, and analytical considerations. Frontiers in Physiology, 4, 34. https://doi.org/10.3389/fphys.2013.00034

Swithers, S. E. (2013). Artificial sweeteners produce the counterintuitive effect of inducing metabolic derangements. Trends in Endocrinology and Metabolism: TEM, 24(9), 431-441. https://doi. org/10.1016/j.tem.2013.05.005
Swithers, S. E., Baker, C. R., \& Davidson, T. L. (2009). General and persistent effects of high-intensity sweeteners on body weight gain and caloric compensation in rats. Behavioral Neuroscience, 123(4), 772-780. https://doi.org/10.1037/a0016139

Swithers, S. E., \& Davidson, T. L. (2008). A role for sweet taste: Calorie predictive relations in energy regulation by rats. Behavioral Neuroscience, 122(1), 161-173. https://doi.org/10.1037/07357044.122.1.161

Swithers, S. E., Martin, A. A., \& Davidson, T. L. (2010). High-intensity sweeteners and energy balance. Physiology \& Behavior, 100(1), 5562. https://doi.org/10.1016/J.PHYSBEH.2009.12.021

Tremmel, M., Gerdtham, U. G., Nilsson, P. M., \& Saha, S. (2017) Economic burden of obesity: A systematic literature review. International Journal of Environmental Research and Public Health, Vol. 14. https://doi.org/10.3390/ijerph14040435

Vigorito, M., \& Sclafani, A. (1988). Ontogeny of polycose and sucrose appetite in neonatal rats. Developmental Psychobiology, 21(5), 457465. https://doi.org/10.1002/dev.420210505

Williams, E. P., Mesidor, M., Winters, K., Dubbert, P. M., \& Wyatt, S. B. (2015). Overweight and Obesity: Prevalence, Consequences, and Causes of a Growing Public Health Problem. Current Obesity Reports, Vol. 4, pp. 363-370. https://doi.org/10.1007/s13679-015-0169-4

Woods, S. C. (1991). The eating paradox: How we tolerate food. Psychological Review, 98(4), 488-505. https://doi.org/10.1037/ 0033-295X.98.4.488

Yeomans, M. R. (2012). Flavour-nutrient learning in humans: An elusive phenomenon? Physiology \& Behavior, 106(3), 345-355. https://doi. org/10.1016/j.physbeh.2012.03.013

Publisher's note Springer Nature remains neutral with regard to jurisdictional claims in published maps and institutional affiliations. 